\title{
Generators of Semigroup and Group
}

\author{
Aloysius Joakim Fernandez \\ Widya mandira Catholic University, Indonesia
}

How to cite this paper: Fernandez, A.J. (2018) Generators of Semi-group and Group. Journal of Applied Mathematics and Coтputation, 2(12), 568-573.

*Corresponding author: Aloysius Joakim Fernandez, Widya mandira Catholic University, Indonesia.

Email: fndz1586@gmail.com

\begin{abstract}
In the journal we will discuss concepts about semigroups and generators from semigroups, as well as properties related to semigroups. Apart from being shown Hille-Yosida's theorem. Next, the dissipative concept will be introduced. In the end, the concepts of the group and its generator will be discussed, as well as the Stone Theorem.
\end{abstract}

Keywords

Semigroup, Generator, dissipative, Group

\section{Introduction.}

In the development of mathematics, many problems can be modeled in a differential equation. This differential equation is then resolved with a strongly continuous semigroups $(T(t))_{t \geq 0}$ on Banach space $X$, as an exponential function operator. Let $B \in \mathcal{L}(X)$ such that it is obtained as follows

$$
T(t)=e^{t B}
$$

for $t \geq 0$.

The purpose of this paper is to provide basic knowledge about Semigroups and Groups. Concepts related to these two topics. More specifically it will be discussed about Semigroup and Group Generators. The theorem related to generators is Hille Yosida's theorem for Semigroups and Stone Theorem for Groups.

\section{Semigroup and Group}

\subsection{SEMIGROUP}

The following are given the concepts of semigrup, which will be used as the basis for the next discussion.

\section{Definition [1]}

A family $(T(t))_{t \geq 0}$ of bounded linear operator in Banach Space $X$ is called Strongly Continuous Semigroup (Semigroup $C_{0}$ ) if it fulfills the following properties:
a. $T(0)=I$
b. $T(t+s)=T(t) T(s)$ for all $t, s \geq 0$
c. Mapping $t \mapsto T(t) x$ is a continuous function from $\mathbb{R}_{+}$to $X$ for all $x \in X$.

\section{Definition [1]}

Let $(T(t))_{t \geq 0}$ is Strongly Continuous Semigroup in Banach Space $\mathrm{X}$ ann let $D(B)$ is a subspace of $X$ which is defined as follows 


$$
D(B):=\left\{x \in X: \lim _{t \searrow 0} \frac{T(t) x-x}{t} \text { exists }\right\}
$$

For all $x \in D(B)$, defined

$$
B x:=\lim _{t \searrow 0} \frac{T(t) x-x}{t}=\left.\frac{d^{+} T(t) x}{d t}\right|_{t=0} .
$$

Operator $B: D(B) \subseteq X \rightarrow X$ is an Infinitesimal Generator of Strongly Continuous Semigroup $(T(t))_{t \geq 0}$. In next dicussion, Infinitesimal Generators will be called Generator. The use of $\lim _{t \backslash 0}$ notation states that positive $t$ value which move down to the value $t=0$.

\section{Theorem [2]}

Let $(T(t))_{t \geq 0}$ be $C_{0}$ semigroup and operator $B$ is Infinitesimal Generator. So

a. For any $x \in X$,

$$
\lim _{l \rightarrow 0} \frac{1}{l} \int_{t}^{t+l} T(h) x d h=T(t) x
$$

b. For any $x \in X, \int_{0}^{t} T(h) x d h \in D(B)$ and

$$
B\left(\int_{0}^{t} T(h) x d h\right)=T(t) x-x
$$

c. For any $x \in D(B), T(t) x \in D(B)$ and

$$
\frac{d}{d t} T(t) x=B T(t) x=T(t) B x
$$

d. For any $x \in D(B)$,

$$
T(t) x-T(h) x=\int_{h}^{t} T(\tau) A x d \tau=\int_{h}^{t} A T(\tau) x d \tau .
$$

\section{Proof}

a. Review

$$
\begin{aligned}
\left\|\frac{1}{l} \int_{t}^{t+l} T(h) x d h-T(t) x\right\| & =\left\|\frac{1}{l} \int_{t}^{t+l}[T(h) x-T(t) x] d h\right\| \\
& \leq \sup _{h \in[t, t+l]}\|T(h) x-T(t) x\| .
\end{aligned}
$$

for $l \rightarrow 0$, caused

$$
\lim _{l \rightarrow 0} \frac{1}{l} \int_{t}^{t+l} T(h) x d h=T(t) x .
$$

b. Let $x \in X, l>0$ and

$$
\lim _{l \rightarrow 0} \frac{T(l)-I}{l}\left(\int_{0}^{t} T(h) x d h\right) \text { exists. }
$$

Noticed

$$
\begin{aligned}
& \frac{T(l)-I}{l}\left(\int_{0}^{t} T(h) x d h\right)=\frac{1}{l} \int_{0}^{t}[T(h+l) x-T(h) x] d h \\
& =\frac{1}{l} \int_{0}^{t} T(h+l) x d h-\frac{1}{l} \int_{0}^{t} T(h) x d h
\end{aligned}
$$




$$
\begin{aligned}
& =\frac{1}{l} \int_{l}^{t+l} T(h) x d h-\frac{1}{l} \int_{0}^{t} T(h) x d h \\
& =\frac{1}{l} \int_{t}^{t+l} T(h) x d h+\frac{1}{l} \int_{l}^{t} T(h) x d h-\frac{1}{l} \int_{0}^{t} T(h) x d h \\
& =\frac{1}{l} \int_{t}^{t+l} T(h) x d h+\frac{1}{l} \int_{h}^{t} T(h) x d h-\frac{1}{l}\left[\int_{0}^{l} T(h) x d h+\int_{l}^{t} T(h) x d h\right] \\
& =\frac{1}{l} \int_{t}^{t+l} T(h) x d h-\frac{1}{l} \int_{0}^{l} T(h) x d h .
\end{aligned}
$$

For $l \rightarrow 0$, obtained

$$
=T(t) x-x .
$$

c. Let $x \in D(B)$ and $k>0$

Will be shown $B T(t) x=T(t) B x$, as follows

Next for $l>0$, obtained

$$
\begin{aligned}
B T(t) x & =\lim _{k \searrow 0} \frac{T(k) T(t) x-T(t) x}{k} \\
& =\lim _{k \searrow 0} T(t) \frac{T(k) x-x}{k} \\
& =T(t) \lim _{k \searrow 0} \frac{T(k) x-x}{k} \\
& =T(t) B x .
\end{aligned}
$$

$$
\begin{aligned}
\frac{d}{d t} T(t) x=\lim _{l \rightarrow 0} \frac{T(t+l) x-T(t) x}{l} & =\lim _{l \rightarrow 0} \frac{T(t) T(l) x-T(t) x}{l} \\
& =\lim _{l \rightarrow 0} T(t) \frac{T(l) x-x}{l} \\
& =T(t) \lim _{l \rightarrow 0} \frac{T(l) x-x}{l} \\
& =T(t) B x \\
& =B T(t) x
\end{aligned}
$$

d. by integrating the results of points (c), obtained

$$
T(t) x-T(h) x=\int_{h}^{t} T(\tau) B x d \tau=\int_{h}^{t} B T(\tau) x d \tau .
$$

\section{Theorem}

Let $B: D(B) \subseteq X \rightarrow X$ be an infinitesimal generatos of strongly continuous semigroup $(T(t))_{t \geq 0}$. Such that $D(B)$ in $X$ and $B$ is closed operator.

\section{Proof}

Let $x \in X, B$ is infinitesimal generator.

Form $x_{t}=\frac{1}{t} \int_{0}^{t} T(s) x d s$

Based on previous theorem (5), $x_{t} \in D(B), t>0$ and based on the previous theorem (4), so it is obtained $x_{t} \rightarrow x$. So that $\overline{D(B)}=X$, On the other hand that $D(B)$ is dense in $X$.

Next will be shown linearity property

For any constants $\eta, \beta$ and $x, y \in X$ 


$$
\begin{aligned}
B(\eta x+\beta y) & =\lim _{t \searrow 0} \frac{T(t)(\eta x+\beta y)-(\eta x+\beta y)}{t} \\
& =\lim _{t \searrow 0} \frac{T(t)(\eta x)+T(t)(\beta y)-\eta x-\beta y}{t} \\
& =\lim _{t \searrow 0} \frac{T(t)(\eta x)-\eta x}{t}+\lim _{t \searrow 0} \frac{T(t)(\beta x)-\beta x}{t} \\
& =\eta B x+\beta B y .
\end{aligned}
$$

Will be shown closure property

Suppose $\left\{w_{n}\right\} \in D(B)$ such that $w_{n} \rightarrow w$ and $B w_{n} \rightarrow y$ for $n \rightarrow \infty$.

Based on (7) Of the previous theorem, obtained

For $n \rightarrow \infty$ obtained

$$
T(t) w_{n}-w_{n}=\int_{0}^{t} T(h) A w_{n} d h
$$

$$
T(t) w-w=\int_{0}^{t} T(h) y d h
$$

Then by dividing the two segments by $t$, for $t \searrow 0$, and based on (a) in the previous theorem that for $w \epsilon D(B)$, then $B w=y$.

\section{Theorem [2]}

A Linear Operator $B$ is infinitesimal generator of contraction semigroup $T(t), t \geq 0$ if and only if

a. $B$ closed dan $\overline{D(B)}=X$.

b. Set of resolvent $\rho(B)$ of $B$ contain $\mathbb{R}^{+}$and for all $\lambda>0$

$$
\|R(\lambda: B)\| \leq \frac{1}{\lambda}
$$

\subsection{GROUP}

\section{Definition [2]}

A family $(T(t))_{t \in \mathbb{R}}$ of bounded linear operator in Banach Space $X$ is called strongly continuous group $\left(C_{0}\right.$ group) if it fulfills following properties
a. $T(0)=I$.
b. $T(t+s)=T(t) T(s)$ for $-\infty<t, s<\infty$.
c. $\lim _{t \rightarrow 0} T(t) x=x$ for $x \in X$.

\section{Definition [2]}

Let $(T(t))_{t \in \mathbb{R}}$ be strongly continuous group in banach Space $X$ nd Let $D(G)$ is subspace of $X$ which is defined as follows

$$
D(G):=\left\{x \in X: \lim _{t \rightarrow 0} \frac{T(t) x-x}{t} \text { exists }\right\} .
$$

For all $x \in D(G)$, defined

$$
G x:=\lim _{t \rightarrow 0} \frac{T(t) x-x}{t} .
$$

Operator $G: D(G) \subseteq X \rightarrow X$ is infinitesimal generator of strongly continuous group $(T(t))_{t \in \mathbb{R}}$.

Given a strongly continuous group $(T(t))_{t \in \mathbb{R}}$ with generator $(G, D(G))$, define $T_{+}(t):=T(t)$ and $T_{-}(t):=T(-t)$ for $t \geq 0$. So $\left(T_{+}(t)\right)_{t \geq 0}$ dan $\left(T_{-}(t)\right)_{t \geq 0}$ are strongly continuous semigroups with each generator $\mathrm{G}$ and $-G$. 
Therefore if $\mathrm{G}$ is generator of group $C_{0}$, so $G$ and $-G$ are generators strongly continuous semigroup. Operator $G$ is a genertor of strongly continuous group $T(t)$ which is defined as follows:

$$
T(t):=\left\{\begin{array}{ll}
T_{+}(t) & t \geq 0 \\
T_{-}(t) & t \leq 0
\end{array} .\right.
$$

Let $X$ is Banach Space and $X^{\prime}$ is its dual. For each $x \in X$, define set of duality $F(x) \subseteq X^{\prime}$ as follows

$$
F(x)=\left\{x^{\prime}: x^{\prime} \in X^{\prime} \text { dan }\left\langle x^{\prime}, x\right\rangle=\|x\|^{2}=\left\|x^{\prime}\right\|^{2}\right\} .
$$

\section{Definition [1]}

A Linear Operator $G: D(G) \subseteq X \rightarrow X$ is dissipative if for each $x \in D(G)$, there is $x^{\prime} \in F(x)$ such that

$$
\operatorname{Re}\left\langle G x, x^{\prime}\right\rangle \leq 0 \text {. }
$$

\section{Theorem [1]}

A linear operator $G: D(G) \subseteq X \rightarrow X$ is dissipative if and only if for each $x \in D(G)$ and $\lambda>0$ it applies

$$
\lambda\|x\| \leq\|(\lambda I-G) x\| \text {. }
$$

\section{Definition [3]}

Let $H$ be Hilbert Space with inner product $\langle$,$\rangle . Operator G: D(G) \subseteq X \rightarrow X$ is called

a. Self-adjoint if $G=G^{*}$

b. Skew-adjoint if $G=-G^{*}$

c. Symetric if $\langle G x, y\rangle=\langle x, G y\rangle$ for each $x, y \in D(G)$

d. Skew-symetric if $\langle G x, y\rangle=-\langle x, G y\rangle$ for each $x, y \in D(G)$.

e.

\section{Theorem [3]}

Let $(G, D(G))$ is a dense operator in Hilbert space $H$. So $G$ generate uniter group $(T(t))_{t \in \mathbb{R}}$ in $H$ if and only if $i G$ self-adjoint, that is $(i G)^{*}=i G$.

\section{Proof}

Suppose that $(G, D(G))$ is a dense operator which generate a uniter group $(T(t))_{t \in \mathbb{R}}$, so for each $x \in D(G)$, obtained

$$
\begin{aligned}
-G x & =\lim _{t \searrow 0} \frac{1}{t}(T(-t) x-x) \\
& =\lim _{t \searrow 0} \frac{1}{t}\left((T(t))^{-1} x-x\right) \\
& =\lim _{t \searrow 0} \frac{1}{t}\left((T(t))^{*} x-x\right) \\
& =G^{*} x .
\end{aligned}
$$

Obtained that $G^{*}=-G$. As a result $(i G)^{*}=-i G^{*}=i G$. So $i G$ is self-adjoint operator.

Instead assume operator on $i G$ self-adjoint, then $G^{*}=-G$. Caused

$$
\langle G x, x\rangle=\left\langle x, G^{*} x\right\rangle=-\langle x, G x\rangle=-\langle\bar{G} x, x\rangle
$$

for all $x \in D(G)=D\left(G^{*}\right)$ and $\langle G x, x\rangle \in i \mathbb{R}$. So $\operatorname{Re}\langle G x, x\rangle=0$ and operator $\mathrm{G}$ is dissipative. Because $G^{*}=-G$ so $\operatorname{Re}\left\langle G^{*} x, x\right\rangle=0$ for $x \in D(G)$. As a result operator $G^{*}$ is dissipative. It is obtained that operator $G$ and $G^{*}=-G$ are infinitesimal generator of semigroups $C_{0}$. If the strongly continuous semigroups $T_{+}(t)$ and $T_{-}(t)$ are generated by operator $G$ and $G^{*}$, which is defined

then $(T(t))_{t \in \mathbb{R}}$ uniter group in $H$.

$$
T(t):= \begin{cases}T_{+}(t) & t \geq 0 \\ T_{-}(t) & t \leq 0\end{cases}
$$




\section{Theorem [2]}

Let $(T(t))$ be semigroup $C_{0}$ of bounded operator. If for all $t>0,(T(t))^{-1}$ is a bounded operator then $S(t)=$ $(T(t))^{-1}$ be semigrup $C_{0}$ of bounded operator which has infinitesimal generator $-G$. Further

$$
U(t)=\left\{\begin{array}{cc}
T(t) & t \geq 0 \\
T(t)^{-1} & t \leq 0
\end{array}\right.
$$

then $U(t)$ is gruop $C_{0}$ of bounded operator.

\section{Proof}

First of all will be shown that $S(t)$ fulfills semigroup properties, as follows

$$
\begin{gathered}
S(0)=T(0)^{-1}=I . \\
S(t+s)=T(t+s)^{-1}=(T(t) T(s))^{-1}=T(t)^{-1} T(s)^{-1}=S(t) S(s) .
\end{gathered}
$$

Next will be shown that $S(t)$ is strongly continuous. For $s>0$, range of $T(s)$ is in $X$. Let $x \in X$ and $s>1$. There is $y \in X$ so that $T(s) y=x$. For $t<1$, obtained

$$
\begin{aligned}
\left\|T(t)^{-1} x-x\right\| & =\left\|T(t)^{-1} T(s) y-T(s) y\right\| \\
& =\left\|T(t)^{-1} T(s) T(t) T(t)^{-1} y-T(s) y\right\| \\
& =\left\|T(t)^{-1} T(t) T(s) T(-t) y-T(s) y\right\| \\
& =\left\|T(t)^{-1} T(t) T(s-t) y-T(s) y\right\| \\
& =\|T(s-t) y-T(s) y\|
\end{aligned}
$$

For $t \searrow 0$, obtained

$$
\|T(s-t) y-T(s) y\| \rightarrow 0 .
$$

Therefore $S(t)$ strongly continuous. For $x \in D(G)$ obtained

$$
\begin{aligned}
\lim _{t \searrow 0} \frac{T(t)^{-1} x-x}{t} & =\lim _{t \searrow 0} T(t) \frac{T(t)^{-1} x-x}{t} \\
& =\lim _{t \searrow 0} \frac{x-T(t) x}{t} \\
& =-G x .
\end{aligned}
$$

then operator $-G$ is infinitesimal generator.

\section{Conclusion}

The infinitesimal generator is an important concept in semigroups and groups. In the semigroup concept, Hille-Yosida Theorem gives characteristics of generator. On the other hand the group concept, Stone theorem gives characteristic to the generator.

This paper contains concepts of semigroup and group, on the other hand this paper can be developed for various solutions to differential equations.

\section{References}

[1] A. Batkai, and S. Piazzera. (2005). Semigroups for Delay Equation, A K Peters Ltd, Canada.

[2] A. Pazy. (1983). Semigroups of Linear Operators and Applications to Partial Differential Equations, Springer-Verlag. New York.

[3] I. I. Vrabie. (2003). $C_{0}-$ Semigroups and Applications. Elsevier .North Hollan.

[4] K-,J. Engel , and R. Nagel. (2000).One-Parameter Semigroup for Linear Evolution Equations. Springer -Verlag. vol.194, Graduate Text in Mathematics. New York. 